\title{
Thymoquinone Prevents Myocardial and Perivascular Fibrosis Induced by Chronic Lipopolysaccharide Exposure in Male Rats
} - Thymoquinone and Cardiac Fibrosis -

\author{
Fereshteh Asgharzadeh ${ }^{1}$, Rahimeh Bargi ${ }^{1}$, Farimah Beheshti ${ }^{4}$, Mahmoud Hos- \\ seini ${ }^{2}$, Mehdi Farzadnia ${ }^{3}$, Majid Khazaei ${ }^{1 *}$
}

'Neurogenic inflammation research center and Department of Physiology, School of Medicine, Mashhad University of Medical Sciences, Mashhad, Iran

${ }^{2}$ Neurocognitive Research Center, School of Medicine, Mashhad University of Medical Sciences, Mashhad, Iran

${ }^{3}$ Departments of Pathology, School of Medicine, Mashhad University of Medical Sciences, Mashhad, Iran

${ }^{4}$ Department of Basic Sciences and Neuroscience Research Center, Torbat Heydariyeh University of Medical Sciences, Torbat Heydariyeh, Iran

\section{Key Words}

thymoquinone, inflammation, cardiac, fibrosis

\begin{abstract}
Objectives: Thymoquinone (TQ) is one of the active ingredients of herbal plants such as Nigella sativa L. (NS) which has beneficial effects on the body. The beneficial effects of TQ on the cardiovascular system have reported. This study aimed to investigate the effect of TQ on cardiac fibrosis and permeability, serum and tissue concentration of inflammatory markers and oxidative stress status in chronic lipopolysaccharide exposure in male rats.
\end{abstract}

Methods: Seventy male Wistar rats were randomly divided into five groups as follows: (1) control; (2) LPS (1 $\mathrm{mg} / \mathrm{kg} /$ day); (3-5) LPS + TQ with three doses of 2, 5 and $10 \mathrm{mg} / \mathrm{kg}$ ( $\mathrm{n}=14$ in each group). After 3 weeks, serum and cardiac levels of IL-1 $\beta$, TNF- $\alpha$ and nitric oxide (NO) metabolites, and cardiac levels of malondialdehyde (MDA), total thiol groups, catalase (CAT) and superoxide dismutase (SOD) activities, permeability of heart

Received: May 09, 2017 Reviewed: May 30, 2018 Accepted: Nov 14, 2018

$(0$ This is an Open-Access article distributed under the terms of the Creative Common Attribution Non-Commercial License (http://creativecommons.org/licenses/by-nc/4.0/) which permits unrestricted noncommercial use, distribution, and reproduction in any medium, provided the original work is properly cited.

( This paper meets the requirements of KS X ISO 9706, ISO 9706-1994 and ANSI/NISO Z39.48-1992 (Permanence of Paper). tissue (evaluated by Evans blue dye method) and myocardial fibrosis were determined, histologically.

Results: LPS administration induced myocardial and perivascular fibrosis and increased cardiac oxidative stress (MDA), inflammatory markers and heart permeability, while, reduced anti-oxidative enzymes (SOD and CAT) and the total thiol group. Administration of TQ significantly attenuated these observations.

Conclusion: TQ improved myocardial and perivascular fibrosis through suppression of chronic inflammation and improving oxidative stress status and can be considered for attenuation of cardiac fibrosis in conditions with chronic low-grade inflammation.

\section{Introduction}

Inflammation is a sort of non-specific immune reaction in response to damage or disease. The acute phase response is a complex systemic initiated by infection, injury, stress, and inflammation. If the replication is not properly staged, in any case, the procedure can form into a subclinical inflammatory state that may trigger distinctive diseases under pathological conditions [1]. Chronic low-grade inflammation or subclinical inflammation is correlated with several chronic

*Corresponding Author

Khazaei Majid. Department of Physiology, Faculty of Medicine, Mashhad University of Medical Sciences, Mashhad, Iran.

Tel: +98-513-800-2227 Fax: +98-38-00-2220

E-mail: khazaeim@mums.ac.ir 
inflammatory diseases such as atherosclerosis, diabetes, and autoimmune diseases [2]. It described by 2-3 fold increment in systemic concentrations of cytokines, such as TNF- $\alpha$, IL-6, and C-reactive protein, and associated with increased oxidative stress markers [3]. However, the exactly related consequences of circulating low dose endotoxemia and the mechanisms especially on the heart tissue are still not clear.

Administration of lipopolysaccharide (LPS) (endotoxin, a component of the cell membrane of gram-negative bacteria) is a known model for the induction of sepsis [4]. It induced a systemic inflammation which has been shown to partially mimic the myocardial and systemic cardiovascular effects of sepsis. LPS depresses myocardial and cardiomyocyte function [5] and induces cardiomyocyte apoptosis [6]. At the point of LPS binding to its receptors, particularly Toll-like receptor (TLR)-4, downstream intracellular signaling pathways are started; a definitive result is the activation of innate immunity [7].

Because of the advancement of treatment complications, for example, drug resistance and adverse effects, traditional medicine are still deficient to provide a complete treatment of specific infections; all things considered, proceeding to discover new medications is expected to give alternative therapy, either to supplement nor supplant existing conventional medicine. The activity of herbal plants contain N. Sativa L. seeds is ascribed to its vital oil part which is predominantly (30-48\%) thymoquinone [8]. Thymoquinone (TQ), with its anti-carcinogenic, anti-inflammatory and anti-oxidant properties has attracted the consideration of researchers to explore its molecular mechanisms and potential use in the treatment of inflammatory diseases [9]. It has several effects on antioxidant enzymes, pro-inflammatory cytokines and some factors involved in cellular signalings such as nuclear factor kappa B or mitogen-activated protein kinase (MAPK) [10]. It also reported that TQ has beneficial effects on the cardiovascular system, respiratory disease, diabetes, hyperlipidemia, and and cancer [9].

In the present study, we used a murine model of recurrent exposure to subclinical LPS and aimed to investigate the effect of TQ on inflammation-induced cardiac fibrosis in chronic low-grade inflammation model in rats.

\section{Material and Methods}

\subsection{Animals and experimental groups}

In this experiment, 70 male Wistar rats, eight weeks old, weighing between 200-250 g, were used. They kept under standard conditions (temperature $22 \pm 2^{\circ} \mathrm{C}$ and 12 hours of light/dark cycle) and free access to food and water. The local ethical committee of animal studies approved the study. The animals randomly divided into five groups that treated as follows: (1) control; (2) LPS; (3-5) LPS+TQ with three doses of 2, 5 and $10 \mathrm{mg} / \mathrm{kg}(\mathrm{n}=14$ in each group).

\subsection{Chemicals}

Lipopolysaccharide, DTNB (5,5' -dithiobis-2- nitrobenzoic acid) and TQ (2-isopropyl-5-methyl-p-benzoquinone) purchased from Sigma (Sigma Chemical Co., USA). LPS dissolved in saline and TQ dissolved in saline supplemented with ethanol 96\%. Tris, TBA (2-thiobarbituric acid), $\mathrm{HCl}$ (Hydrochloric acid), EDTA (Ethylene di amine tetra acetic acid), TCA (Trichloro acetic acid), DMSO (5,5-dithio-bis-(2-nitrobenzoic acid), diethyl ether, formamide and Evans blue obtained from Merck (Merck Co., Germany).

\subsection{Study design}

For induction of chronic low-grade inflammation, LPS groups received $1 \mathrm{mg} / \mathrm{kg} /$ day, intraperitoneally (ip), for three weeks $[11,12]$. The animals of the control group received saline instead of LPS. TQ-treated groups were received TQ (2, 5 or $10 \mathrm{mg} / \mathrm{kg} /$ day; ip) from the beginning of the study [13]. After three weeks, the animals anesthetized with diethyl ether and blood samples were taken from orbital sinus for biochemical analysis. The blood samples centrifuged at $3000 \mathrm{rpm}$ for 20 minutes, and serums were collected and stored at $-70^{\circ} \mathrm{C}$ for further analysis. Then, half of the animals in each group sacrificed. The hearts were isolated, washed with saline and the left ventricles used for histopathological assessment. The right ventricles were also dissected to homogenize for determination of tissue oxidative, anti-oxidative, nitrite and inflammatory markers. In the rest of animals, the permeability of the heart tissues evaluated as described below.

\subsection{Measurement of serum and tissue levels of TNF- $\alpha$ and IL-1 $\beta$}

The specific rat ELISA kits (ebioscience Co, San Diego, CA, USA) used for serum and heart homogenate TNF- $\alpha$ and IL- $1 \beta$ measurements according to the manufactory instructions.

\subsection{Serum and tissue levels of nitric oxide metab- olite}

For the measurement of serum and heart nitrite, Griess reagent method was used (Promega Corp, USA) [14]. In brief, sulfanilamide and NED solutions put in room temperature. First, $50 \mu \mathrm{l}$ of the sample (serum or tissue homogenate) added to the wells. Then, $50 \mu \mathrm{l}$ of sulfanilamide solution added to all samples into the wells and incubated for 5-10 minutes at room and away from light. Next, NED solution poured to all samples. At this stage, color purple appeared. Optical densities of samples were measured using a microplate reader at wavelength 520-550 $\mathrm{nm}$. The mean optical densities of each sample obtained by the reading device by using the standard curve nitrite concentrations. 
A

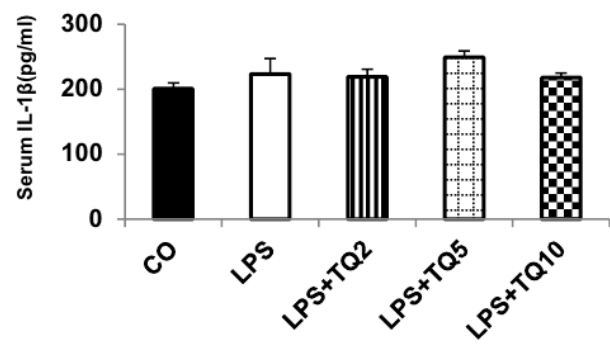

C

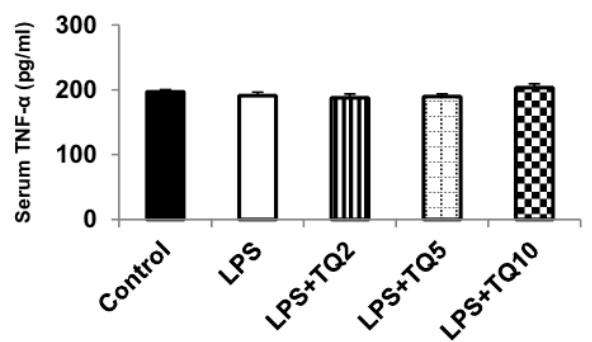

B

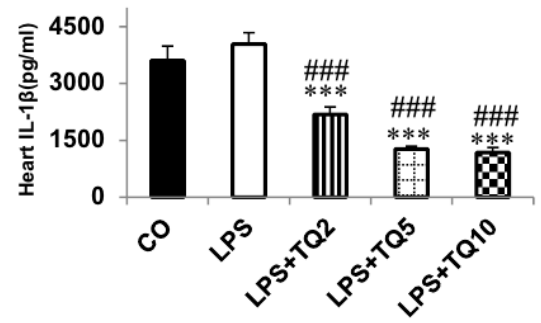

D

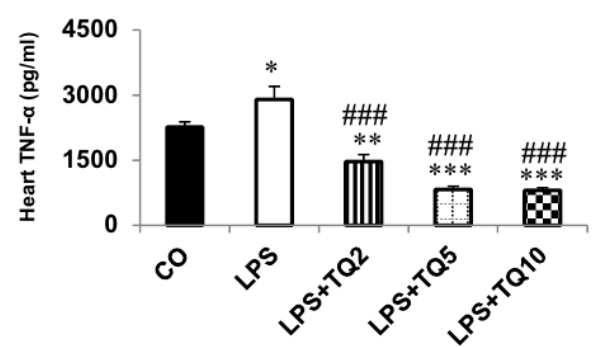

Figure 1 Serum (A) and heart (B) IL-1 $\beta$ levels in experimental groups. ${ }^{* * *} \mathrm{p}<0.001$ compare to control group and \#\#\#p $<$ 0.001 compare to LPS group. Changes of Serum (C) and heart (D) TNF- $\alpha$ level in experimental groups. ${ }^{* * *} \mathrm{p}<0.001,{ }^{* *} \mathrm{p}<$ $0.01,{ }^{*} \mathrm{p}<0.05$ compare to control group and \#\#\# $<0.001$ compare to LPS group. $\mathrm{n}=10$ in each group.

A

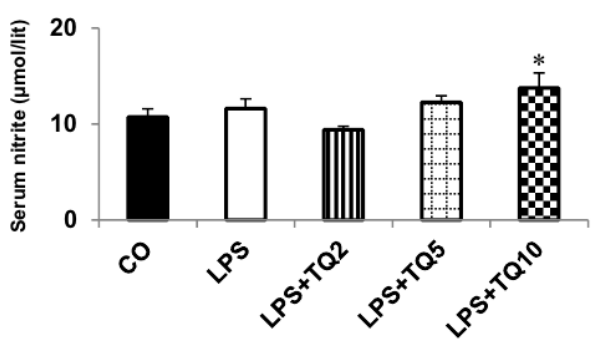

B

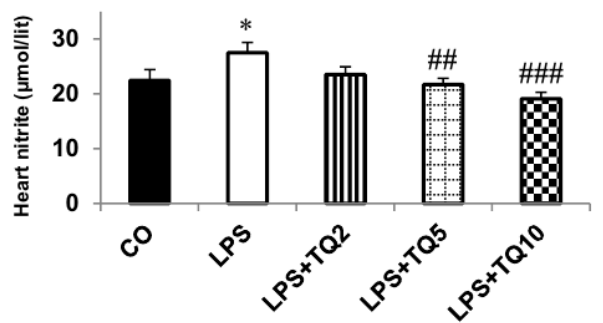

Figure 2 Serum (A) and heart (B) nitrite concentrations. ${ }^{*} \mathrm{p}<0.05$ compare to control group, \#\#\# $<0.001$ and \#\#p $<0.01$ compare to LPS group. $\mathrm{n}=10$ in each group. $0.01,{ }^{*} \mathrm{p}<0.05$ compare to control group and \#\#\#p $<0.001$ compare to LPS group. $\mathrm{n}=10$ in each group.

\subsection{Measurement of malondialdehyde (MDA)}

MDA level is an index of lipid peroxidation. For its evaluation, the target tissue was weighed and homogenized immediately with a phosphate buffer solution. Then, $1 \mathrm{ml}$ of $10 \%$ homogenates with two mL of TBA + TCA + HCL solution mixed for 45 minutes in a boiling water bath. After cooling to room temperature, they centrifuged for $10 \mathrm{~min}$ utes. Then, the absorbance at $535 \mathrm{~nm}$ read and MDA was calculated based on the following formula [15].

$$
\mathrm{C}(\mathrm{M})=\mathrm{A} / 1.65 \times 10^{5}
$$

\subsection{Measurement of total thiol groups (SH)}

To measure the total thiol group, DTNB (Di-Tio nitro benzoic acid) reagent used. This represents the yellow color complex reacts with SH groups. $1 \mathrm{ml}$ of Tris-EDTA buffer $(\mathrm{pH}=8.6)$ added to tissue homogenate and the specimen absorbance measured at $412 \mathrm{~nm}$ against Tris-EDTA buffer alone (A1). Next, $20 \mu \mathrm{l}$ of DTNB reagents were added to the solution and kept at room temperature for 15 minutes. Then, the sample absorbance read again (A2). The absorbance of DTNB reagent was read alone as a blank (B). Total thiol concentration $(\mathrm{mM})$ calculated from the following 
formula [16].

Total thiol concentration $(\mathrm{mM})$

$=(\mathrm{A} 2-\mathrm{A} 1-\mathrm{B}) \times 1.07 / 0.05 \times 13.6$

\subsection{Measurement of superoxide dismutase and catalase activity}

Superoxide dismutase (SOD) was measured using a Ransod kit (Randox Laboratory, UK). Catalase action was measured by a method as previously described (Aebi, 1984). In brief, the principle of this method determined on the hydrolyzation of $\mathrm{H} 2 \mathrm{O} 2$ in phosphate buffer, $\mathrm{pH}$ 7.0, and requisite volume of sample and decreasing absorbance at $240 \mathrm{~nm}$. The Conversion of $\mathrm{H} 2 \mathrm{O} 2$ into $\mathrm{H} 2 \mathrm{O}$ and $\mathrm{O} 2$ in 1 minute under standard condition was thought to be the enzyme response velocity.

\subsection{Heart permeability measurement}

Heart permeability was measured by quantifying the Evans blue (EB) dye bound to albumin. The animals were anesthetized , and EB dye $(20 \mathrm{mg} / \mathrm{ml})$ injected through the tail vein before sacrifice. After 20 minutes, the hearts were dissected, weighted, and placed in $4 \mathrm{~mL}$ formamide solution for 48 hours at room temperature. The optical density of the solution was measured by spectrophotometry at 620 $\mathrm{nm}$. Data expressed as $\mu \mathrm{g} \mathrm{EB/g}$ of wet weight tissue [17].

\subsection{Histopathological assessment}

The left ventricles placed in $10 \%$ buffered formalin for 24 hours preceding preparing and paraffin taking after a standard system. At that point, serially segmented into $5 \mu \mathrm{m}$ thick samples were set up from the tissue pieces, stained with hematoxylin and eosin (H\&E) and Masson's trichrome and analyzed under light microscopy. The area of collagen content (blue stained) surrounding the coronary artery was also measured and normalized to the control group.

\subsection{Statistical analysis}

Data expressed as the mean \pm standard error of the mean (SEM). All data were analyzed using the SPSS 20 software. The comparisons between groups were evaluated using one-way ANOVA, followed by LSD posthoc test. The level of significance considered if $\mathrm{p}<0.05$.

\section{Results}

\subsection{Body weight}

The results showed that LPS group had lower body weight gain compared to control, although it was not statistically significant ( $15.4 \pm 3.6$ vs. $20.2 \pm 6.41 \mathrm{~g}$, respectively). Weight gain in the animals who received TQ with doses of 2, 5 and $10 \mathrm{mg} / \mathrm{kg}$ was: $4.2 \pm 1.08,3.5 \pm 0.42$ and $1.6 \pm 0.45$; respectively.

\subsection{Serum and cardiac inflammatory marker levels}

Measurement of serum levels of IL-1 $\beta$ (Fig. 1A) and TNF- $\alpha$ (Fig. 1B) showed that there were no significant differences between LPS and control groups and treatment by TQ couldn't alter them ( $p>0.050)$. Evaluation of cardiac levels of IL- $1 \beta$ and TNF- $\alpha$ showed that animals in the LPS group had higher levels than control. Administration of TQ by all three doses $(2,5$ and $10 \mathrm{mg} / \mathrm{kg})$ lowered serum levels of IL- $1 \beta$ compare to the LPS group ( $p<0.001)$. The same results were observed in cardiac TNF- $\alpha$ after treatment by TQ. TQ dose-dependently reduced tissue levels of IL-6 and TNF- $\alpha$ (Fig. 1C\&D).

\subsection{Serum and cardiac NO metabolite}

As shown in figure 2A, there was no significant difference in serum nitrite concentration between LPS and control groups ( $p>0.05)$. Administration of TQ by three doses could not significantly change serum nitrite level ( $p>0.05)$. However, cardiac level of nitrite in the LPS group showed higher level compare to control group $(\mathrm{p}<$ 0.05 ). Treatment by TQ reduced the level of cardiac nitrite, dose-dependently (Fig. 2B), without changing in serum nitrite level.

\subsection{Oxidant and antioxidant markers in cardiac tissue}

Figure 3A\&B illustrate that LPS group had higher MDA concentration (Fig. 3A) and lower total thiol contents (Fig. 3B) in cardiac tissues compare to control animals ( $\mathrm{p}<$ 0.001 ). Treatment by TQ with all doses decreased cardiac MDA and increased total thiol concentration $(p<0.001)$. In addition, LPS administration decreased cardiac SOD (Fig. 3C) and catalase (Fig. 3D) activity compare to control animals $(\mathrm{p}<0.001)$. Administration of TQ increased catalase and SOD activity, dose dependently.

\subsection{Heart permeability}

Results showed that heart permeability of LPS animals was higher than control animals $(\mathrm{p}<0.001)$. All doses of TQ decreased cardiac permeability $(\mathrm{p}<0.001)$ which reached to control level by high dose of TQ ( $<<0.001)$ (Fig. 4).

\subsection{Histopathological evaluation}

The H\&E stained histopathological examination illustrated that there were no pathological changes in ventricular muscle structure in the control group (Fig. 5A). In contrast, in LPS group, increased infiltration of inflammatory cells and disarrangement of myofibers were observed (Fig. 5B). Administration of TQ illustrated improvement of pathological changes, dose dependently (Fig. 5C-E).

Staining with Masson's trichrome illustrated that in LPS administration significantly increased in collagen deposition in myocardium compared to control rats (Fig. 6A\&B). 
A

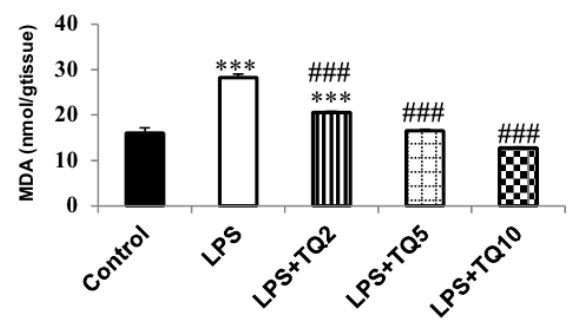

C

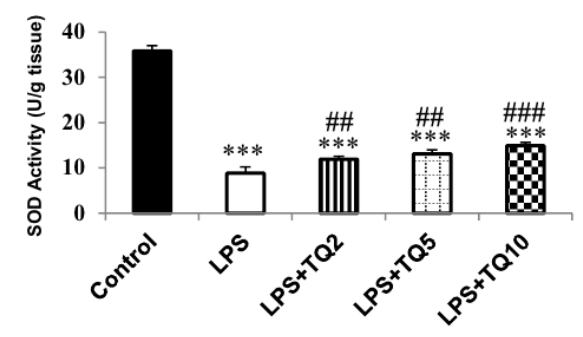

B

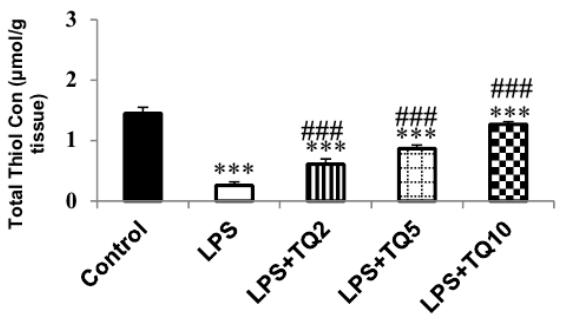

D

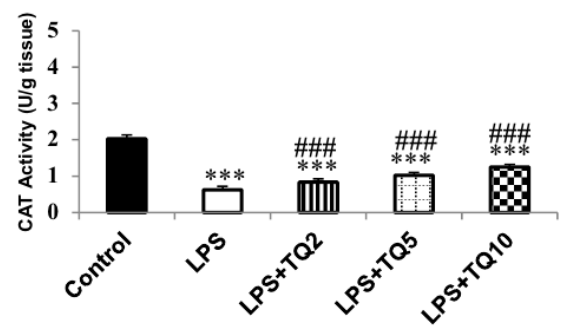

Figure 3 Comparison of MDA (A) and total thiol concentration (B) in heart homogenate in experimental groups. ${ }^{* * *}$ p < 0.001 compare to control group and \#\#\#p $<0.001$ compare to LPS group. SOD (C) and catalase (D) activities in heart tissue. ${ }^{* * *} \mathrm{p}<0.001,{ }^{* *} \mathrm{p}<0.01$ compare to control group; \#\#\# $<0.001$, \#\#p $<0.01$ compare to LPS group. $\mathrm{n}=10$ in each group.
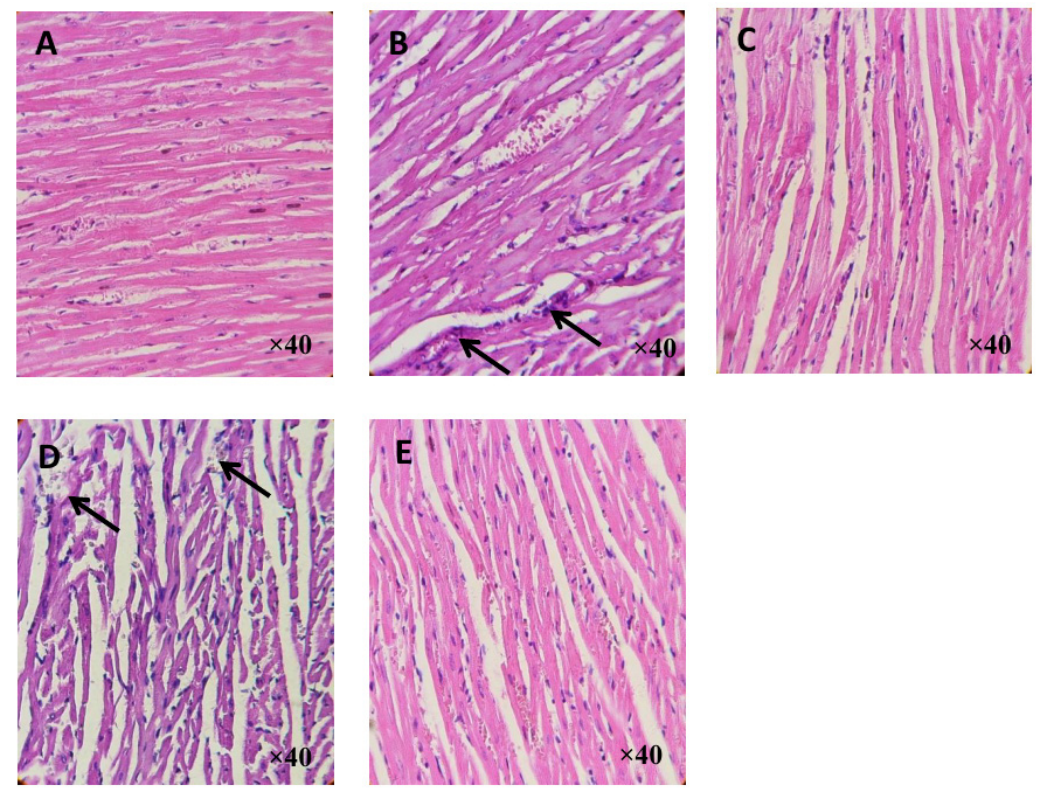

Figure 5 H\&E staining of left ventricles in control (A) and LPS (B) groups. Arrows indicate infiltration of inflammatory cells and disarrangement of fibers. TQ reduced infiltration of inflammatory cells in the heart tissue (C-E: TQ 2, 5, and 10 $\mathrm{mg} / \mathrm{kg}$; respectively). $\mathrm{n}=6$ in each group. 


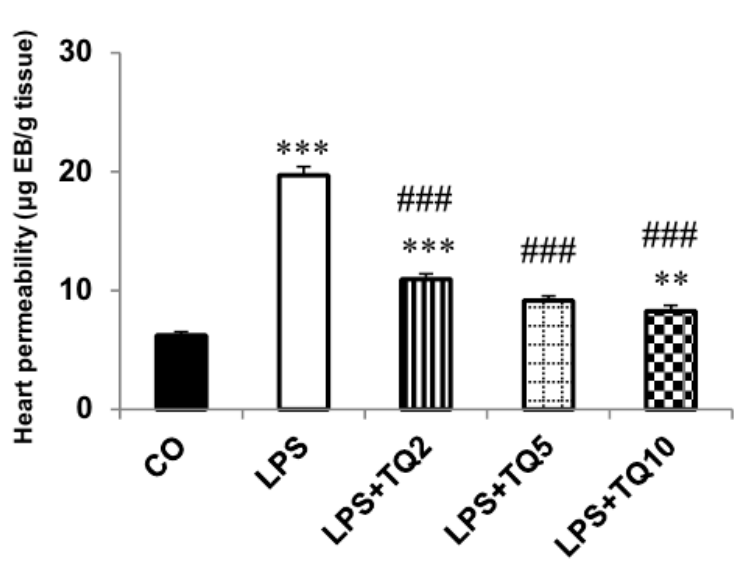

Figure 4 Permeability of heart tissue in experimental groups. ${ }^{* *} \mathrm{p}<0.001,{ }^{* *} \mathrm{p}<0.01$ compare to control group; $\# \#$ p $<0.001$ compare to LPS group. $n=7$ in each group.

TQ induced as dose dependent decrease in cardiac fibrosis (Fig. 6C-E). Also, the increment in percent of collagen deposition in LPS group was observed compared to the control group (Fig. 6F; p < 0.001). Administration of TQ (all doses) reduced the percent of collagen deposition in the left ventricular wall $(\mathrm{p}<0.001)$. Also, chronic LPS exposure resulted in a significant perivascular collagen deposition around coronary artery which prevented by TQ treatment (Fig. 7).

\section{Discussion}

Our results showed that Also exposure to chronic inflammation induced by LPS, cardiac fibrosis occurs as a primary effect. And TQ reduced it. TQ has beneficial effects on cardiovascular health, and its anti-inflammatory and antioxidant activity examined in both in vitro and in vivo models [10]. In this study, the effect of TQ on cardiac fibrosis and permeability and serum and tissue concentrations of inflammatory markers and oxidative stress in the subclinical LPS-induced model of inflammation studied. In this study, chronic injection of LPS did not change body weight compared to the control group. Wilbur et al. reported that no change in activity, appetite , and body weight observed in rats that exposed to LPS injection [18]. In our study, administration of TQ (all doses) in LPS group significantly reduced body weight. It showed that treatment with TQ has positive effects in reduction of body weight and metabolic syndrome factors [19].

Our study showed that the heart levels of IL-1 $\beta$ and TNF- $\alpha$ were significantly higher in the LPS group which was in line with previous studies [20]. Administration of TQ reduced tissue levels of the inflammatory cytokines, dose-dependently. The ability of TQ in reducing serum levels of inflammatory cytokines such has observed in other inflammatory models [21]. Since inflammation process is regulated lipoxygenase and cyclooxygenase, TQ plays as anti-inflammatory through inhibition of cyclooxygenase and 5-lipoxygenase membrane [22]. The production of inflammatory mediators such as IL- $1 \beta$, TNF- $\alpha$, NO and
PGE2 is set mainly by NFKB (as regulators key of genes involved in inflammatory responses) [23]. In the present study, tissue levels of nitrite in the LPS group were higher than control and treatment by TQ decreased tissue level of NO significantly. It suggested that TQ decreased NO generation by reducing the expression of iNOS [24]. Mahmoudi et al. showed that TQ, dose-dependently, reduced NO production in the supernatant of stimulated macrophages by LPS, as well as inhibited an increase in mRNA expression of inducible nitric oxide [25]. Subclinical inflammation also is associated with increased oxidative stress [26]. NFKB transcription factor regulates the expression of inflammatory mediators that involved in oxidative stress [27], thus inhibiting the production of NFKB can prevent the events resulting in injury. The production of reactive oxygen species induced by LPS causes a wide variety of cellular responses by activating transcription factor NFKB through MAPKs and PI3K / Akt routes [28]. It indicated that TQ inhibits these transcription factors and thus reduce inflammation and oxidative stress [8]. In the present study, chronic injection of LPS accompanied with a significant increase in the concentration of MDA, lower content of thiol groups and reduced activity of SOD and catalase in the heart tissue. Interestingly, TQ reduced oxidative stress markers and increased anti-oxidative parameters in LPS groups which support the previous studies [8].

The morphological changes in endothelial cells by bacterial infections cause increased its permeability and endothelial dysfunction [29]. We observed a significant increase in permeability of heart tissue in LPS animal and injection of TQ reduced heart permeability, dose-dependently Studies have also specified that NFKB has an significant role in endothelial response to inflammatory stress [30]. Thus, it is possible that TQ improves endothelial barrier function which damaged by LPS via strong antioxidant activity possibly through its ability to inhibit NFKB. Excessive collagen by fibrocytes changed the ventricular walls structures and interferes with the normal function of the heart, as a result of increased fibrosis and heart failure [31]. The level of subclinical LPS causes cardiac fibrosis that may associated with the development of cardiovascular disease [18], and studies have shown that inflammation is a risk factor for cardiac fibrosis. It also specified that the activation of ROS might lead to cardiac fibrosis [32]. Results of the present study showed cardiac fibrosis (indicated by more collagen content) in chronic inflammation induced by LPS [2]. Interestingly, TQ improved left the ventricular wall and perivascular fibrosis as dose-dependent manner. It suggested that NFKB activation leads to the expression of inflammatory markers and fibrotic factors [33] and it is possible that TQ could inhibit this pathway resulted in the anti-fibrotic and antioxidant effect of TQ.

\section{Conclusion}

In conclusion, exposure to the chronic low-grade level of LPS is accompanied by fibrosis in the myocardium and perivascular tissue, heart permeability, and oxidative stress status. TQ attenuated these observations which suggest being considered in treatment in conditions with chronic low-grade inflammation. 

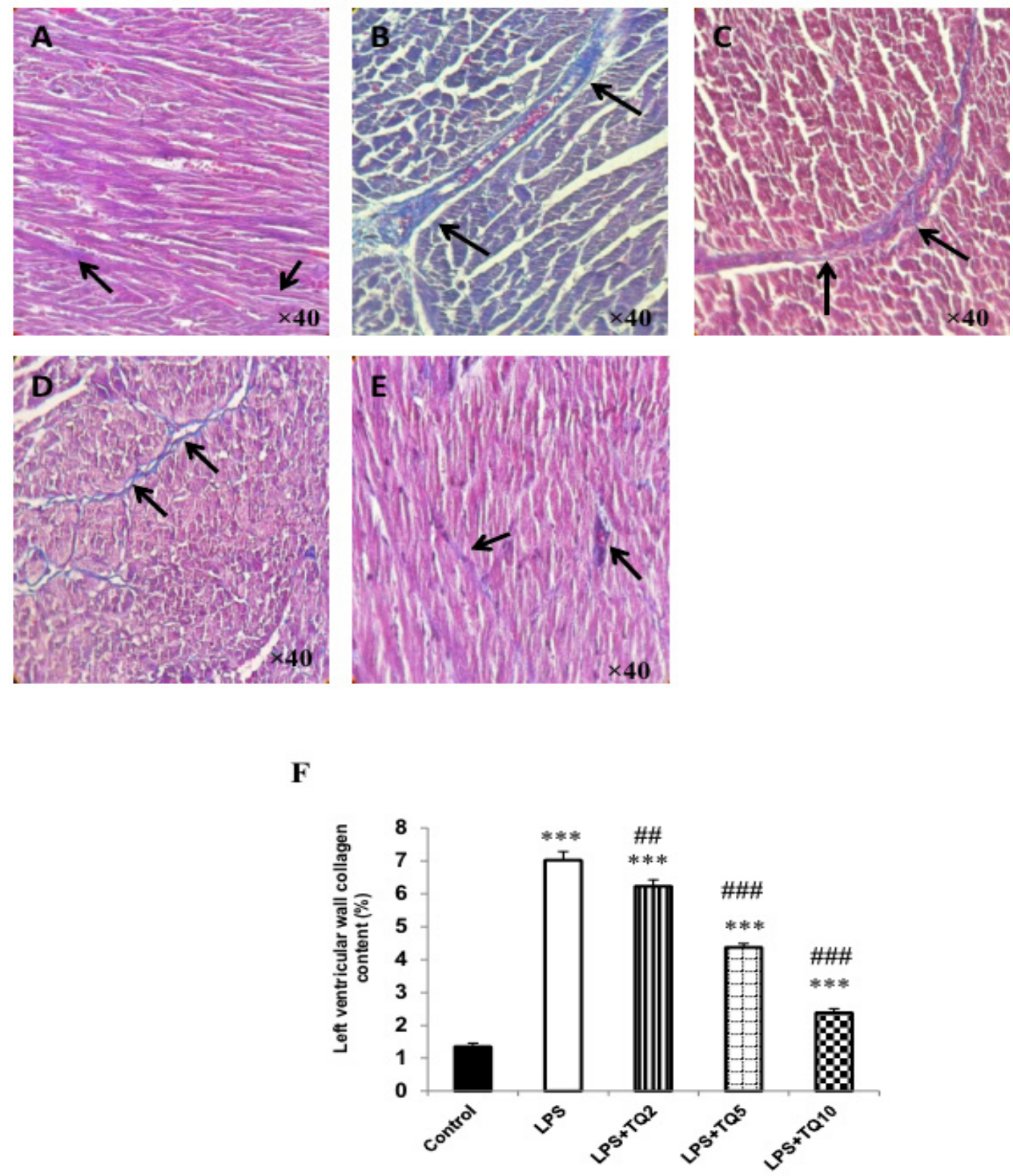

Figure 6 Masson trichrome staining of left ventricular wall fibrosis. A: control; B: LPS group shows more collagen deposition (Blue color indicates collagen fibers characterized by black arrow). Administration of TQ improved cardiac fibrosis dose dependently. Cardiac fibrosis expressed as higher collagen content (\%) in LPS group compare to control which improved by TQ administration (F). ${ }^{* * *} \mathrm{p}<0.001$ compare to control group; \#\#\#p $<0.001$ and \#\#p $<0.01$ compare to LPS group. $\mathrm{n}=6$ in each group. 

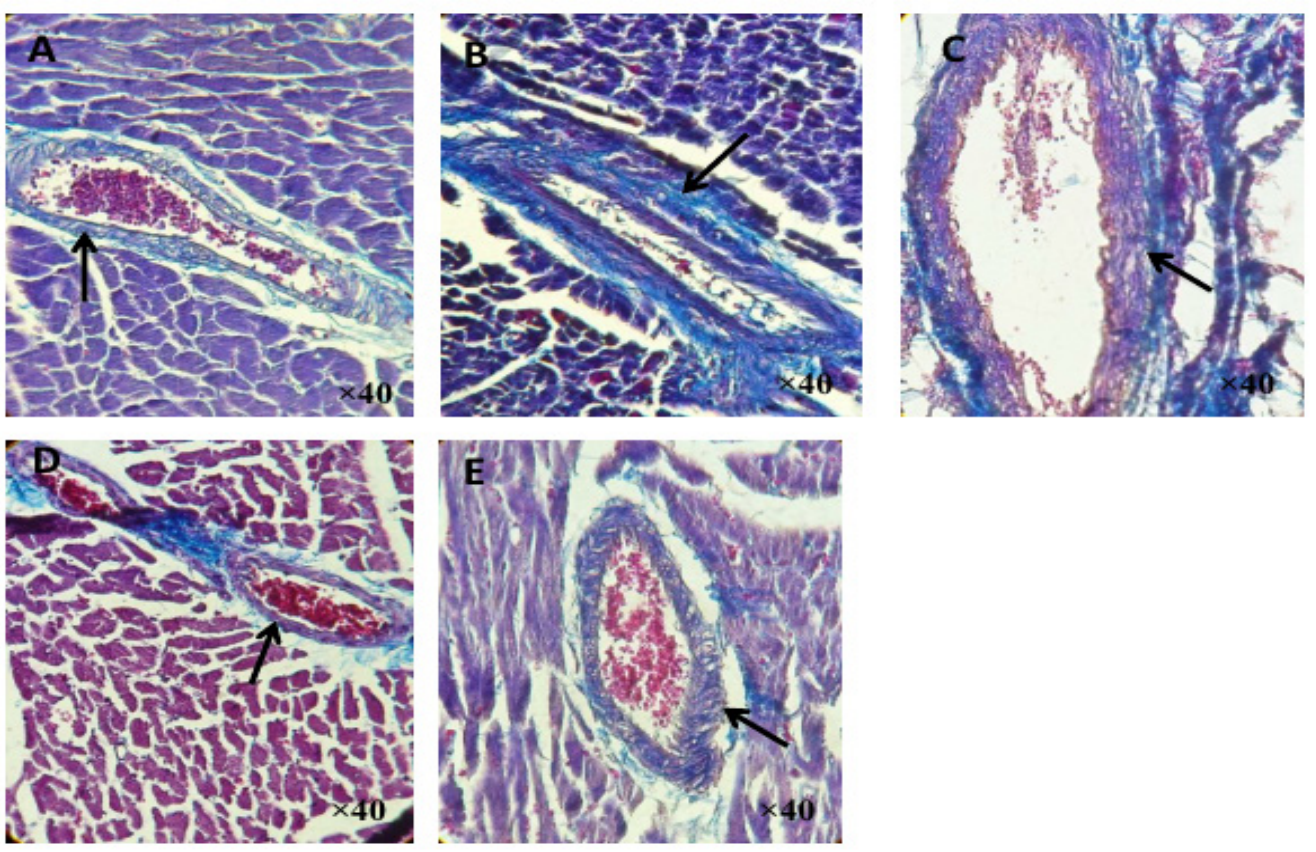

F

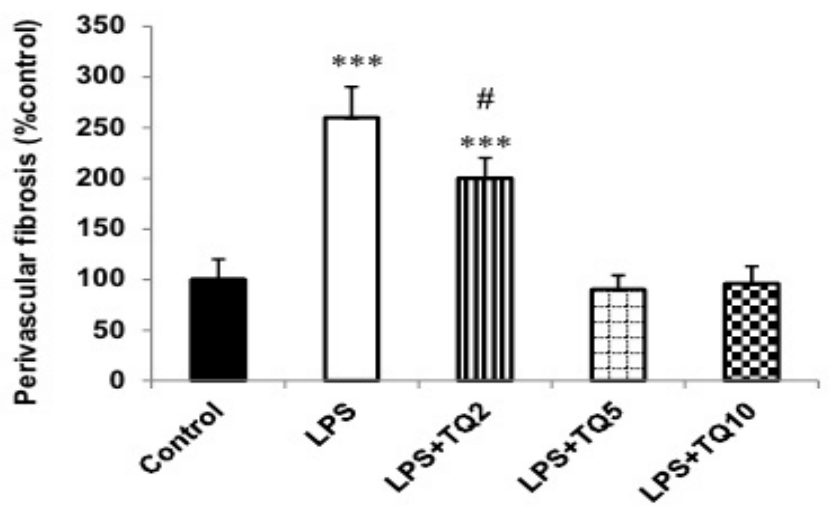

Figure 7 Perivascular fibrosis around left anterior descending coronary artery (black arrows) stained by Masson trichrome. Blue color indicates collagen deposition. A: control; B: LPS; C-E: TQ by doses of 2, 5 and $10 \mathrm{mg} / \mathrm{kg}$; respectively. \#: $\mathrm{p}<0.05$ compare to LPS; ${ }^{* * *}$ : $\mathrm{p}<0.001$ compare to control. 


\section{Acknowledgment}

The authors are thankful for vice chancellor of Mashhad University of Medical Sciences for their financial support (grant number: 931641).

\section{Conflict of interest}

The authors declare that there are no conflicts of interest.

\section{References}

1. Ahmad I, Muneer KM, Tamimi IA, Chang ME, Ata MO, Yusuf N. Thymoquinone suppresses metastasis of melanoma cells by inhibition of NLRP3 inflammasome. Toxicol Appl Pharmacol. 2013;270(1):70-6.

2. Alimohammadi S, Hobbenaghi R, Javanbakht J, Kheradmand D, Mortezaee R, Tavakoli M, et al. Protective and antidiabetic effects of extract from Nigella sativa on blood glucose concentrations against streptozotocin (STZ)-induced diabetic in rats: an experimental study with histopathological evaluation. Diagn Pathol. 2013;8:137.

3. Asgharzadeh F, Rouzbahani, R, Khazaei, M. Chronic low-grade inflammation: Etiology and its effects Journal of Isfahan Medical School. 2016;34(379):408-21.

4. Bai T, Lian LH, Wu YL, Wan Y, Nan JX. Thymoquinone attenuates liver fibrosis via PI3K and TLR4 signaling pathways in activated hepatic stellate cells. Int Immunopharmacol. 2013;15(2):275-81.

5. Bargi R, Asgharzadeh F, Beheshti F, Hosseini M, Sadeghnia HR, Khazaei M. The effects of thymoquinone on hippocampal cytokine level, brain oxidative stress status and memory deficits induced by lipopolysaccharide in rats. Cytokine. 2017;96:173-84.

6. Darakhshan S, Bidmeshki Pour A, Hosseinzadeh Colagar A, Sisakhtnezhad S. Thymoquinone and its therapeutic potentials. Pharmacol Res. 2015;95-96:138-58.

7. Doi K, Leelahavanichkul A, Yuen PS, Star RA. Animal models of sepsis and sepsis-induced kidney injury. J Clin Invest. 2009;119(10):2868-78.

8. El-Mahmoudy A, Matsuyama H, Borgan MA, Shimizu Y, El-Sayed MG, Minamoto N, et al. Thymoquinone suppresses expression of inducible nitric oxide synthase in rat macrophages. Int Immunopharmacol. 2002;2(11):1603-11.

9. Ghazwani M, Zhang Y, Gao X, Fan J, Li J, Li S. Anti-fibrotic effect of thymoquinone on hepatic stellate cells. Phytomedicine. 2014;21(3):254-60.

10. Gholamnezhad Z, Havakhah S, Boskabady MH. Preclinical and clinical effects of Nigella sativa and its constituent, thymoquinone: A review. J Ethnopharmacol. 2016;190:372-86.

11. Hayden MS, Ghosh S. Signaling to NF-kappaB. Genes Dev. 2004;18(18):2195-224.

12. Helmersson J, Vessby B, Larsson A, Basu S. Association of type 2 diabetes with cyclooxygenase-mediated inflammation and oxidative stress in an elderly population. Circulation. 2004;109(14):1729-34.

13. Hosseinzadeh H, Sadeghnia HR. Safranal, a constitu- ent of Crocus sativus (saffron), attenuated cerebral ischemia induced oxidative damage in rat hippocampus. J Pharm Pharm Sci. 2005;8(3):394-9.

14. Nematollahi S, Nematbakhsh M, Haghjooyjavanmard S, Khazaei M, Salehi M. Inducible nitric oxide synthase modulates angiogenesis in ischemic hindlimb of rat. Biomed Pap Med Fac Univ Palacky Olomouc Czech Repub. 2009;153(2):125-9.

15. Hotamisligil GS, Erbay E. Nutrient sensing and inflammation in metabolic diseases. Nat Rev Immunol. 2008;8(12):923-34.

16. Janero DR. Malondialdehyde and thiobarbituric acid-reactivity as diagnostic indices of lipid peroxidation and peroxidative tissue injury. Free Radic Biol Med. 1990;9(6):515-40.

17. Khazaei M, Nematbakhsh M. Coronary vascular and aortic endothelial permeability during estrogen therapy: a study in DOCA-salt hypertensive ovariectomized rats. Physiol Res. 2004;53(6):609-14.

18. Ko HJ, Oh SK, Jin JH, Son KH, Kim HP. Inhibition of Experimental Systemic Inflammation (Septic Inflammation) and Chronic Bronchitis by New Phytoformula BL Containing Broussonetia papyrifera and Lonicera japonica. Biomol Ther (Seoul). 2013;21(1):66-71.

19. Leon CG, Tory R, Jia J, Sivak O, Wasan KM. Discovery and development of toll-like receptor 4 (TLR4) antagonists: a new paradigm for treating sepsis and other diseases. Pharm Res. 2008;25(8):1751-61.

20. Lew WY, Bayna E, Molle ED, Dalton ND, Lai NC, Bhargava V, et al. Recurrent exposure to subclinical lipopolysaccharide increases mortality and induces cardiac fibrosis in mice. PLoS One. 2013;8(4):e61057.

21. Li Q, Verma IM. NF-kappaB regulation in the immune system. Nat Rev Immunol. 2002;2(10):725-34.

22. Mansour M, Tornhamre S. Inhibition of 5-lipoxygenase and leukotriene C4 synthase in human blood cells by thymoquinone. J Enzyme Inhib Med Chem. 2004;19(5):431-6.

23. Tahergorabi Z, Khazaei M. The relationship between inflammatory markers, angiogenesis, and obesity. ARYA Atheroscler. 2013;9(4):247-53.

24. Mehta PK, Griendling KK. Angiotensin II cell signaling: physiological and pathological effects in the cardiovascular system. Am J Physiol Cell Physiol. 2007;292(1):C82-97.

25. Min HY, Song SH, Lee B, Kim S, Lee SK. Inhibition of lipopolysaccharide-induced nitric oxide production by antofine and its analogues in RAW 264.7 macrophage cells. Chem Biodivers. 2010;7(2):409-14.

26. Norouzi F, Abareshi A, Asgharzadeh F, Beheshti F, Hosseini M, Farzadnia M, et al. The effect of Nigella sativa on inflammation-induced myocardial fibrosis in male rats. Res Pharm Sci. 2017;12(1):74-81.

27. Petersen AM, Pedersen BK. The anti-inflammatory effect of exercise. J Appl Physiol (1985). 2005;98(4):115462.

28. Elmi S, Sallam NA, Rahman MM, Teng X, Hunter AL, Moien-Afshari F, et al. Sulfaphenazole treatment restores endothelium-dependent vasodilation in diabetic mice. Vascul Pharmacol. 2008;48(1):1-8.

29. Sallam N, Khazaei M, Laher I. Effect of moderate-inten- 
sity exercise on plasma C-reactive protein and aortic endothelial function in type 2 diabetic mice. Mediators Inflamm. 2010;149678(10):2.

30. Tekeoglu I, Dogan A, Demiralp L. Effects of thymoquinone (volatile oil of black cumin) on rheumatoid arthritis in rat models. Phytother Res. 2006;20(10):869-71.

31. Wang Y, Gao H, Zhang W, Fang L. Thymoquinone inhibits lipopolysaccharide-induced inflammatory mediators in BV2 microglial cells. Int Immunopharmacol. 2015;26(1):169-73.

32. Woo CC, Kumar AP, Sethi G, Tan KH. Thymoquinone: potential cure for inflammatory disorders and cancer. Biochem Pharmacol. 2012;83(4):443-51.

33. Yasuda S, Lew WY. Lipopolysaccharide depresses cardiac contractility and beta-adrenergic contractile response by decreasing myofilament response to $\mathrm{Ca} 2+$ in cardiac myocytes. Circ Res. 1997;81(6):1011-20. 